\title{
A Flow Visualization Study of Spore Release Using a Wind Tunnel-Mounted Laser Light Sheet
}

J. M. Davis, Department of Marine, Earth \& Atmospheric Sciences, and Department of Plant Pathology, North Carolina State University, Raleigh, 27695-8208; A. D. Eisner, ManTech Environmental Technology, Inc., P.O. Box 12313, Research Triangle Park, NC 27709; R. W. Wiener, Atmospheric Chemistry and Physics Branch, EPA National Exposure Research Laboratory, Research Triangle Park, NC 27711; and C. E. Main, Department of Plant Pathology, North Carolina State University, Raleigh 27695-7616

\begin{abstract}
Davis, J. M., Eisner, A. D., Wiener, R. W., and Main, C. E. 1997. A flow visualization study of spore release using a wind tunnel-mounted laser light sheet. Plant Dis. 81:1057-1065.

A phase Doppler anemometry system in combination with a laser light sheet was used in a lowspeed recirculating wind tunnel to examine the flow field around an individual leaf. Turbulence similar to that encountered near the surface of the earth in a neutral stability boundary layer was generated using a grid at the upwind end of the wind tunnel test section. Individual healthy and diseased plant leaves were introduced into the tunnel with the leaf tip pointing downwind. The Mie-scattered radiation from the spores departing the diseased leaf was captured on videotape. Image processing software was used to enhance the visual quality of the individual frames from the videotape and to make spore velocity calculations. Three main vortex regions around the leaf were identified. The importance of these regions to the separation of the spores from the leaf surface and their subsequent downwind movement was analyzed.
\end{abstract}

Additional keywords: coherent structures, sporangiospores

Fundamental research has been conducted on both the long- and short-distance atmospheric transport and biometeorology of fungal sporangiospores (2,5-9). The early papers (7) concentrated on long-distance transport and involved the use of the Atmospheric Transport and Dispersion (ATAD) model (12). This model uses observed upper air wind fields to advect spore clouds according to the observed averaged spatial/temporal wind field. An improved version of the ATAD model, known as the Branching Atmospheric Trajectory (BAT) model, was later developed to account for the diurnal structural changes that occur in the atmospheric boundary layer (13). The HY-SPLIT model (10) is the newest version available and is currently being used to provide real-time forecasts of spore cloud movement as part of an agricultural meteorology extension service. Forecast boundary layer winds are operationally provided by the National Weather Service numerical weather prediction models. Short-range (10 to $15 \mathrm{~km}$ ) transport of sporangiospores has also been examined using numerical analysis schemes (29). The objective of this study

Corresponding author: Jerry M. Davis

E-mail: davis@climate.nrrc.ncsu.edu

ordavis@niss.rti.org

Accepted for publication 2 June 1997.

Publication no. D-1997-0714-02R

(C) 1997 The American Phytopathological Society was to examine the first stage in the spore transport process, i.e., the release and the movement of spores from the leaf surface.

The sporangiospores of Peronospora tabacina were used for all wind tunnel experimental work because they possess unique optical properties. $P$. tabacina is a classical downy mildew pathogen, i.e., sporulation occurs on the ventral leaf surface. These spores are produced on a dichotomously branched sporangiophore. Spore release occurs primarily by mechanical processes such as the rubbing of leaves together as a result of wind forces or the twisting of the sporangiophore resulting from stalk drying as the vapor pressure gradient between the stalk and the environment increases $(15,22)$. The majority of the liberated spores never leave the crop canopy (2). Of those that do, a large number travel only a short distance before impaction. However, a significant number of spores is entrained into the lowest portions of the atmospheric boundary layer. It is these spores that are eventually mixed throughout the boundary layer and are transported to near and distant locations, thereby spreading the pathogen to new potential disease sites.

The main research objective was to examine the turbulent flow field around healthy and diseased plant leaves using laser light sheet flow visualization techniques and phase Doppler anemometry (PDA). The flow visualization procedures used allow one to record on the videotape (or some other medium) the movement of the spores away from the underside of a diseased leaf. Using image-capturing technology and digital image analysis of the video frames, the vortex structures that arise on both sides of diseased and healthy leaves can be viewed. With the use of a time series of video frames, one can calculate the flow characteristics in the vicinity of the leaf.

\section{MATERIALS AND METHODS}

Wind tunnel physical characteristics. A low-velocity recirculating wind tunnel with a counterclockwise flow regime was used in this research $(19,27)$. In plan view, the tunnel is rectangular in shape with approximate outside dimensions of 4.9 by $18.1 \mathrm{~m}$, which yields a centerline circuit around the tunnel of $46 \mathrm{~m}$. The cross-sectional area of the tunnel (which was constructed using English units of measurement) in the test section is about $1.85 \mathrm{~m}^{2}$ $\left(20 \mathrm{ft}^{2}\right)$. The width of the test section is

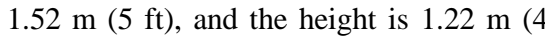
$\mathrm{ft}$ ). The tunnel is capable of operating at speeds up to about $13 \mathrm{~m} \mathrm{~s}^{-1}$ ( $\left.29 \mathrm{mph}\right)$. When the tunnel is operated at high wind speeds, a significant amount of heat is added to the recirculating airstream. A bypass chiller/blower system is used to control the temperature in the tunnel, which allows the tunnel to operate at a temperature near ambient.

Operation at low wind speeds (between 0.5 and $2.0 \mathrm{~m} \mathrm{~s}^{-1}$ ) was required in this research. This was achieved by operating only the chiller/blower system at a reduced flow rate. The tunnel also has a bank of medium efficiency bag-type filters downstream of the test section to remove aerosol (spores) from the recirculating airstream.

The tunnel was used to provide a controlled environment in which the flow patterns around plant leaves could be investigated. Because of the fundamental operating objective, it was desirable to have uniform flow across the test section where the leaf would be mounted. Initially, low turbulence intensity flow was achieved using common garden lattice. At $2 \mathrm{~m}$ upstream from the entrance to the effuser (contraction) section of the wind tunnel, a metal honeycomb grid is permanently mounted. The cells are $10-\mathrm{cm}$ squares that are $61 \mathrm{~cm}$ deep. Common garden lattice was placed against the upwind side of this 
honeycomb. Both the permanent grid and the garden lattice act to reduce the turbulence intensity in the wind tunnel. They accomplish this reduction by removing the large-scale eddies at the expense of introducing a larger number of smaller scale eddies that rapidly decay. At short downwind distances from the grid pair, turbulence intensities may be considerably increased, but at points further downwind the turbulence is decreased. When the grid pair is placed at the entry point to the contraction section of the tunnel, the eddies have more time to decay before reaching the main portion of the test section. In addition, the contraction accelerates the mean flow, thus decreasing the turbulence intensity. The effect of the grid pair on the turbulence and velocity distributions appears to depend mainly on a pressure drop coefficient. See Pankhurst and Holder (19) for an extended discussion on the effects of grids on wind tunnel turbulence. The actual test measurements will be discussed in the results section. This uniformity of flow was considered necessary for the turbulence tripping experimentation that followed. Of primary importance to the project was the use of a full-size, naturally sporulating leaf. Because of geometric similarity requirements, the use of a fullsize leaf precluded modeling the neutral stability atmospheric boundary layer in this wind tunnel. However, since the leaf in its natural setting is exposed to various levels of turbulence intensity, it was still desirable to simulate the natural characteristics of the environment and to make the properties of the generated turbulence as close to those found in the lower atmosphere (as indicated by the spectra of the longitudinal wind component, u) as possible $(14,20$, 21,24,25).

The spectrum of the longitudinal wind component $(\mathrm{u})$ from the wind tunnel was compared with spectra obtained from micrometeorological experiments. These spectra covered the entire frequency range and take the form:

$$
\frac{f S(f)}{\sigma_{u}^{2}}=\frac{N}{(1+1.5 N)^{5 / 3}}
$$

where $N$ is nondimensional frequency $\left(f \lambda_{m} / \bar{u}\right), \quad \lambda_{m}$ is the wavelength corresponding to the largest eddy, $f$ is frequency, $S(f)$ is the spectrum of the longitudinal wind component $(u), \bar{u}$ is the mean longitudinal wind speed, and $\sigma_{u}^{2}$ is the variance of the longitudinal wind component. In the current setting, the mean wind speed was $0.5 \mathrm{~m} \mathrm{~s}^{-1}$, and the wavelength of the largest eddy was taken to be equal to the opening dimension $(0.5 \mathrm{~m})$ in the turbulence tripper.

Turbulence in the wind tunnel was generated by a biplanar grid positioned at the downwind end of the contraction section, i.e., at the entry point of the test section. The grid had one vertical and one horizontal cross member, each $25.4 \mathrm{~cm}$ wide.
There were four open areas, each $43.2 \mathrm{~cm}$ high and $58.4 \mathrm{~cm}$ across. The wake turbulence from a number of grid configurations was examined before the sizes were selected. The selection of a grid configuration was based on the spectra and turbulence intensities that would be found in the surface layer portion of the atmospheric boundary layer.

Hot-wire anemometry and phase Doppler anemometry. A hot-wire anemometer was used to characterize the air flow in the wind tunnel $(3,11)$. The probe was attached to a rigid horizontal tube which was supported by two carriages mounted on an optical bench. The carriages could slide on the bench with high precision and stability as necessary in hotwire applications. The probe was inserted into the test section through small openings in the tunnel wall and moved perpendicularly to the wall in order to conduct measurements several points and several elevations in the test section. Thermal anemometers measure air velocity by sensing the changes in heat transfer from a small, electrically heated sensor exposed to the air motion. The standard probe sensing element is a small piece of wire or a thin film of metal, which is heated by an electrical current. Changes in the air flow cause a change in the electrical current. In the present application, air temperature, composition, and pressure could be considered constant so that the only variable affecting heat transfer was air velocity.

Laser Doppler anemometry (LDA) allows for the measurement of particle velocities by detecting the Doppler frequency shift of laser light that has been scattered by small particles moving with the fluid (30). Phase Doppler anemometry (PDA) is an extension of LDA that allows for the simultaneous measurement of the size and velocity of individual particles in complex two-phase flow. PDA is based on the assumptions that the particles to be sized are spherical and traverse a uniform field of plane waves, and that the scattering angle is selected such that a single mode of scattering dominates the scattered light collected by the receiving optics.

Flow visualization. The flow visualization method used in this study incorporated a laser light sheet, light scattering particles, a video-recording camera, and image capturing/digitizing hardware and software. A video imaging board capable of interactive image digitizing and analysis was used. Light scattering particles were introduced into the flow either by smoke-wire techniques, as described below, or by using naturally released spores from the plants in the test section of the tunnel. The light sheet was generated by launching the beam from a fixed-position argon ion laser into a single fiber-optic cable terminated with a miniature collimator. The laser beam that emerged from the collimator was $1.5 \mathrm{~mm}$ in diameter and was delivered to a spinning mirror assembly located under the floor of the wind tunnel. The mirror assembly was composed of a DC motor capable of $18,000 \mathrm{rpm}$ and a laser-quality mounted mirror attached to the motor axis. A slit in the floor of the tunnel allowed the beam to enter the tunnel test section and illuminate the underside of the leaf. The slit was covered with a clear 1.5 -mm-thick Plexiglas strip. A mirror placed on the inside top of the tunnel over the leaf provided laser illumination for the top of the leaf surface. Aerosol particles introduced into the flow regime interacted with the laser light to produce Mie scattering (26). A portion of the scattered light was detected and recorded on VHS tape using the video camera.

The optical properties of sporangiospores make them ideal for flow visualization research. Liquid water is a major constituent of the interior of the spore. The radiation extinction coefficient for pure water reaches its lowest value in the blue portion of the visible wavelength band. The laser light sheet from the argon ion laser was centered in the near-green portion of the spectrum; thus reflectivity values should be high.

The spore is a complex optical structure. Some portion of the radiation impinging on the spore will be reflected from the outer wall of the spore and never enter the spore itself. To penetrate to the center of the spore, radiation must pass through the double wall that surrounds each spore. The trapping of some portion of the radiation between the two walls is likely. Radiation will be refracted as it penetrates through the walls. Multiple internal reflections will occur inside the spore. Some absorption of the radiation will occur at each contact point during the reflection process. However, some of the radiation will undergo forward scattering and eventually leave the spore and arrive at the video camera to be collected. Diffraction around the edge of the spore will also occur. The size of the spore relative to the wavelength of the impinging radiation and the spore's transparent material make viewing possible.

The smoke-wire technique (which does not use smoke produced by combustion, but rather a fog produced by nucleation and then condensation of a supersaturated vapor) employs a variety of aerosol-generating compounds. The technique $(4,28)$ is capable of producing very fine smoke particles and can be used to study the detailed structure of complex flow phenomenon. Smoke particles are small enough to follow the air flow with precision. Therefore, in cases where mixing is not strong enough to produce a uniform concentration of smoke particles, various quasi-steady flow structures can be observed by illuminating the smoke particles with a light sheet. Smoke curls and other smoke structures described below are actually quasi-steady features of the flow. In our setup, the smoke was pro- 
duced by vaporizing the liquid used in theatrical smoke generators. The liquid was applied by a constant feed technique (which employed an adjustable automated syringe pump) to two fine steel wires that had been twisted together. The twisted wire was stretched vertically in the middle of the tunnel upwind from the test section. AC current, controlled by a Variac, was applied to the wire to make it sufficiently hot to vaporize drops of liquid sliding downward on its surface. Within millimeters of the wire, the vapor would condense to produce smoke. The average size of the smoke drops was 1 to $2 \mu \mathrm{m}$ in diameter. The twisted wires produced smoke of high density, with discrete streaks of smoke resulting from periodically sliding drops and the liquid trapped in the cavities of the twisted wires. For a video camera, highquality images require the discrete streaks to produce high levels of contrast with a dark background.

Plant inoculation and spore trapping. Tobacco plants of the cultivar Burley 21 were grown in the North Carolina State University Phytotron greenhouse until the 10-leaf stage. They were then placed in an environmentally controlled growth chamber, and each leaf was inoculated with sporangiospores of $P$. tabacina (isolate PT91 Weaverville). The plants were kept in the dark under atomized moisture for 24 $\mathrm{h}$, after which they were exposed to fluorescent and incandescent light (approximately $4.3 \times 10^{4}$ lux) for $9 \mathrm{~h}$ each day for 7 days. As disease symptoms appeared (yellow lesions), the plants were returned to complete darkness for $24 \mathrm{~h}$ to induce sporulation. Abundant, fresh spores were present at the start of the wind tunnel experiment.

In the wind tunnel, greased slides and a rotorod spore sampler were used to capture spores leaving the underside of the diseased leaf. The greased slides were placed on the tunnel floor at $50-\mathrm{cm}$ intervals from a point directly under the leaf to a point 3 $\mathrm{m}$ downwind from the plant. The rotorod sampler was placed $60 \mathrm{~cm}$ downwind from the leaf. Only a few individual spores were collected on the slides; however, the rotorod collected many spores. Microscope analysis indicated that individual spores were leaving the leaf, not spore clusters.

Terminal settling velocity. The terminal settling velocity for $P$. tabacina sporangiospores was estimated in an unpublished preliminary study. A settling tower was constructed from black PVC pipe (20 $\mathrm{cm}$ diameter by $166 \mathrm{~cm}$ tall) fitted midway with two windows (7- by $15-\mathrm{cm}$ optical glass) perpendicular to each other to permit videotaping of falling spores at right angles to a laser light sheet. The vertically orientated light sheet was generated by a 10 $\mathrm{mW}$ helium-neon laser equipped with a $30^{\circ}$ fan-type beam splitter. A video camera was equipped with a 55-mm, 1:28 lens to visualize images of the falling spores. Spores (isolate PT91Y collected in Yancey Co., NC, in 1991) were released from infected leaves attached to the underside of the sealed lid of the settling tower. All experiments were conducted at room temperature $\left(22^{\circ} \mathrm{C}\right)$ and relative humidity $(70 \%)$. Videotape images of the falling spores were analyzed electronically with commercially available software equipped with a frame grabber board to provide the graphical interface.

Characterization of wind tunnel turbulence. Before the main experimental work could be initiated, the basic turbulence characteristics in the wind tunnel test section had to be determined. A hot-wire probe was used to sample the mean flow and turbulence intensity for the longitudinal velocity component (u) on a 30-point grid established in the part of the test sec-
A
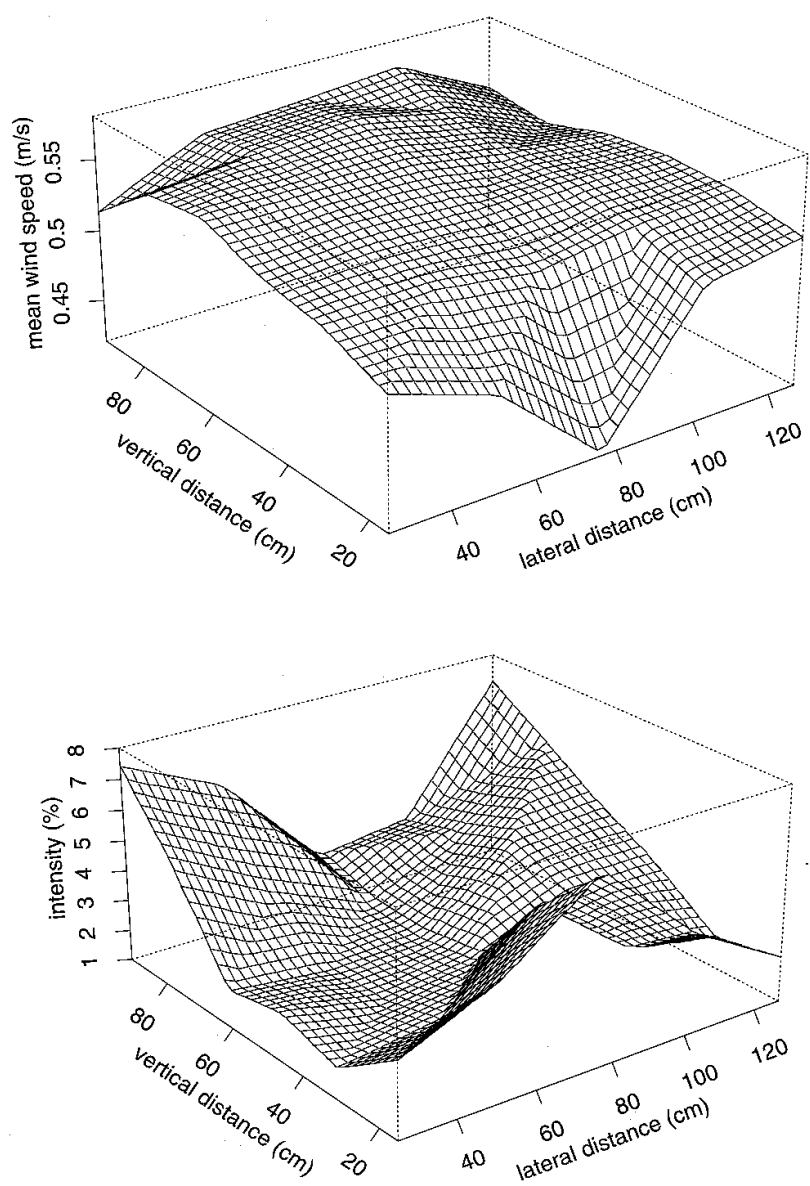

B

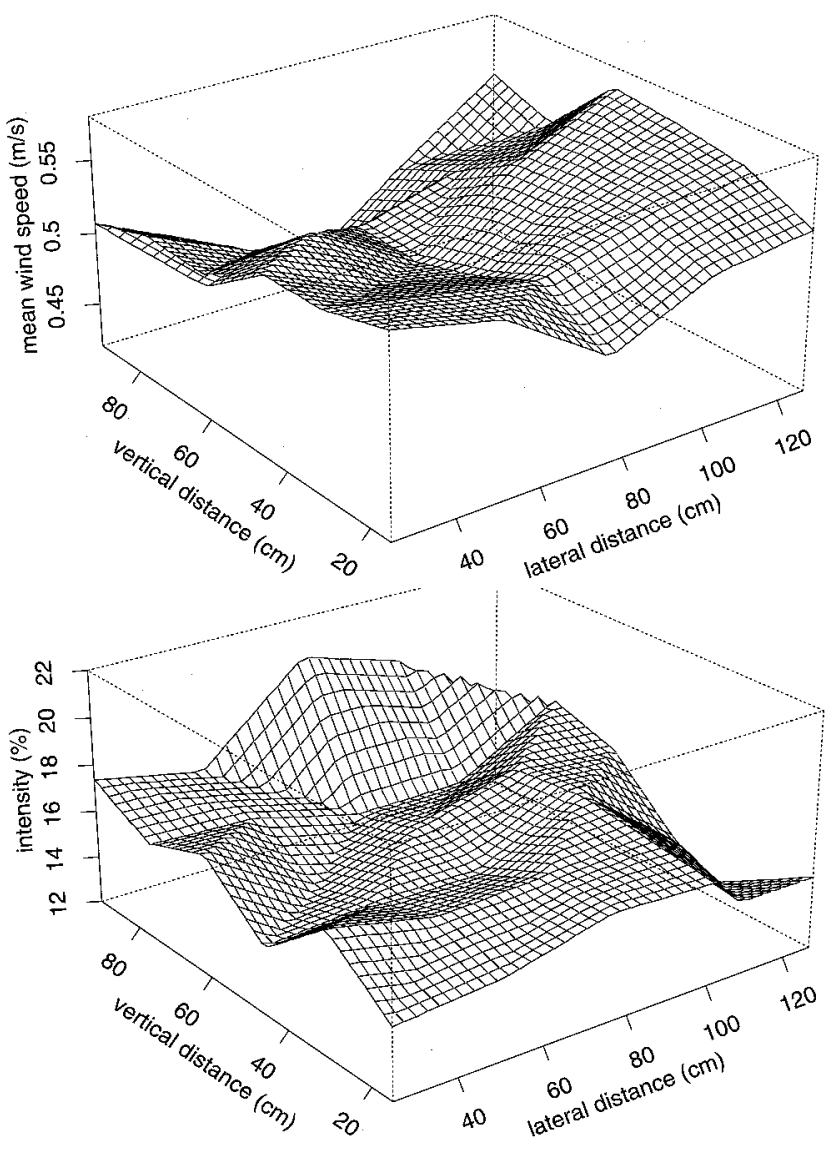

Fig. 1. (A) A perspective view of the wind tunnel mean wind speed $\left(\mathrm{m} \mathrm{s}^{-1}\right)$ and turbulence intensity $(\%)$ when the turbulence tripper was not in the tunnel. Grid points were in the $y($ lateral)-z(vertical) plane and were located in that portion of the test section where the leaf was to be placed. (B) Same except with the turbulence tripper in place. 
tion where the major portion of the experimental work was to be done. In the vertical direction, there were six points, the first of which was located $15.2 \mathrm{~cm}$ (6 in) off the tunnel floor, and subsequent points were located $15.2 \mathrm{~cm}$ apart. The top point was $30.5 \mathrm{~cm}$ (12 in) below the top of the tunnel test section. There were five points in the lateral (cross tunnel) direction. The first and last points were each $25.4 \mathrm{~cm} \mathrm{(10}$ in) from the sides of the test section.

Figure 1 shows a perspective view of the flow characteristics measured on the grid mentioned above. The tunnel was set to operate at approximately $0.5 \mathrm{~m} \mathrm{~s}^{-1}$. Figure 1A shows flow conditions without the turbulence tripper in place. In the center of the tunnel test section, where the leaf was eventually located, both the mean wind speed and the turbulence intensity were found to be uniform. The speed was about $0.54 \mathrm{~m} \mathrm{~s}^{-1}$ (with a standard deviation of about $0.02 \mathrm{~m} \mathrm{~s}^{-1}$ ), and the turbulence intensity was about $3 \%$ (with a standard deviation of about 1\%). This flow closely approximates that found in laminar conditions. Near the tunnel walls, the wind speeds are lower because of the boundary layer effect, and the turbulence intensities are higher. Figure 1B shows conditions when the turbulence tripper is in place. In the center of the tunnel, wind speeds were near $0.51 \mathrm{~m} \mathrm{~s}^{-1}$ (with a standard deviation of about $\left.0.03 \mathrm{~m} \mathrm{~s}^{-1}\right)$, and turbulence inten-

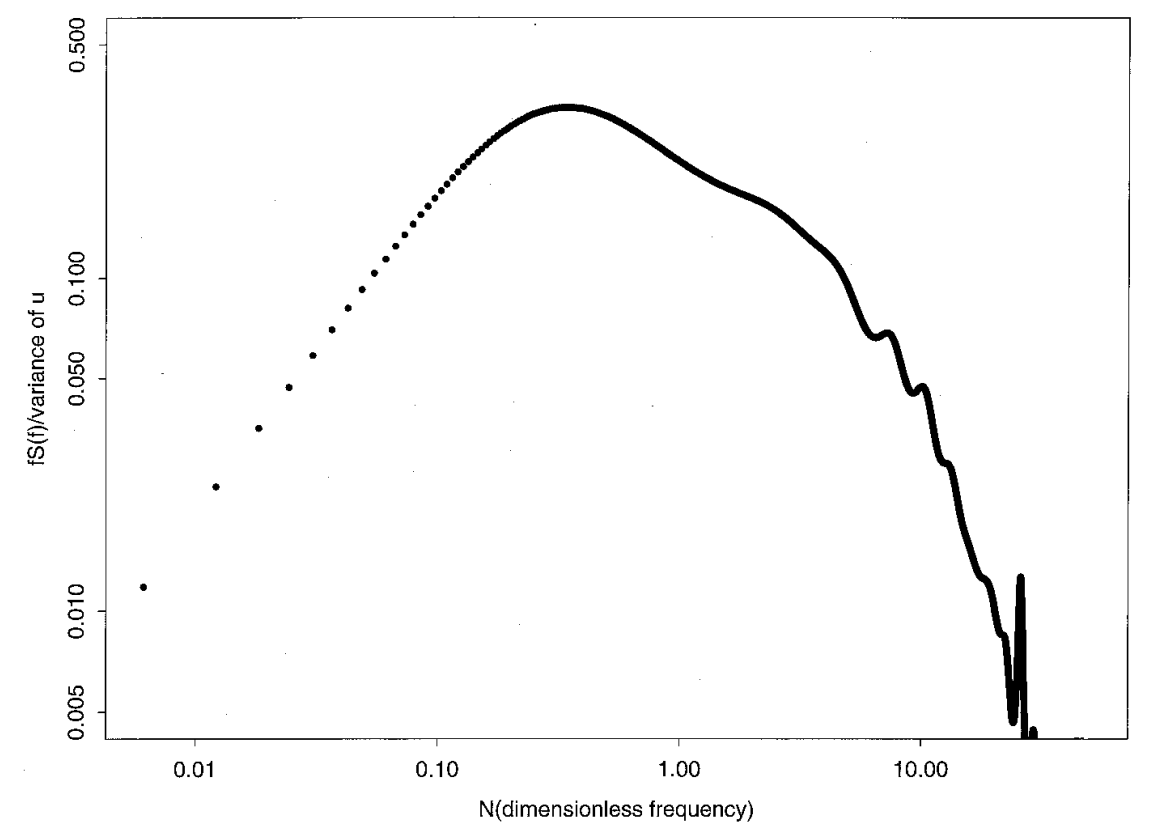

Fig. 2. The power spectrum for the longitudinal (u) velocity component measured at the $y-z$ center point in that portion of the test section where the leaf was to be placed. The spectrum coordinates selected are the same as those used in the boundary layer meteorological literature (see text).

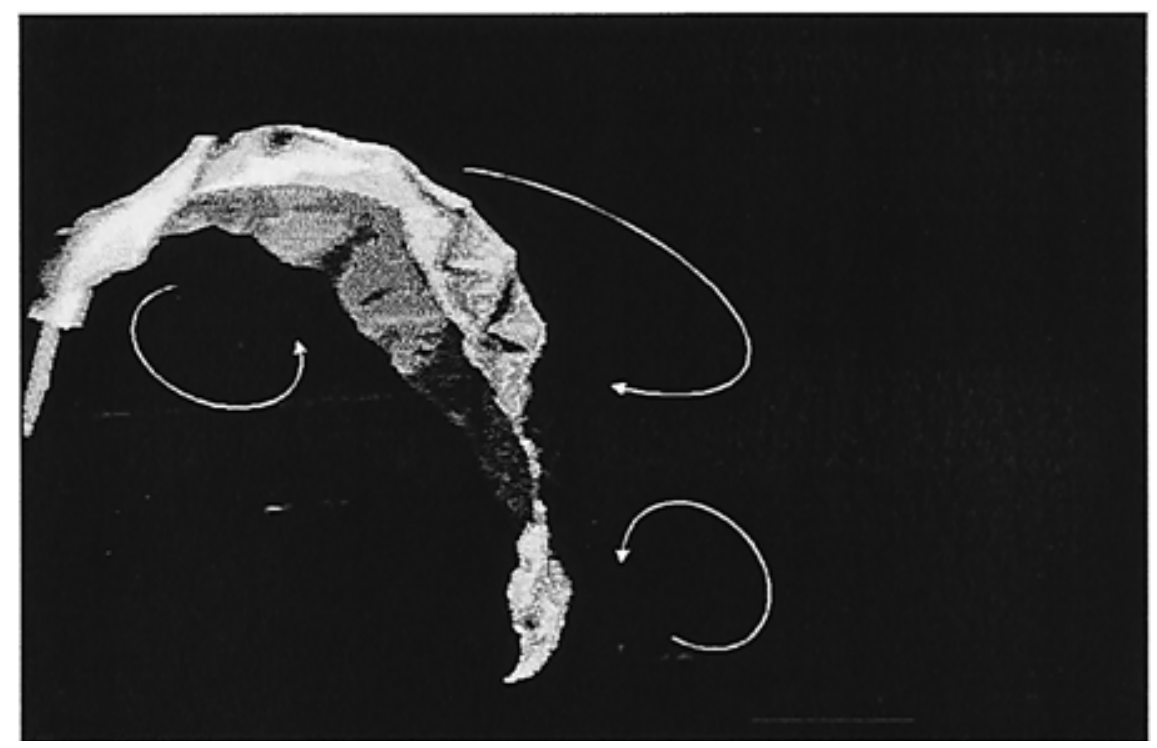

Fig. 3. Diagram of a diseased leaf with the main vortex regions indicated.

sities were around $16 \%$ (with a standard deviation of about $1 \%$ ), a value consistent with conditions near the earth's surface.

As mentioned earlier, one of the objectives with regard to the turbulence produced with the tripper was to match as closely as possible the structure of turbulence in the neutral atmospheric boundary layer (14). Figure 2 shows the results of the measurement made in the middle (in the $\mathrm{y}$ and $\mathrm{z}$ directions) of the tunnel test section at the point where leaf measurements were usually taken. Measurements were taken with the tunnel set to operate at $0.5 \mathrm{~m} \mathrm{~s}^{-1}$. The actual mean wind speed at this location was $0.49 \mathrm{~m} \mathrm{~s}^{-1}$, and the turbulence intensity was $16 \%$. The data sampling rate was $0.1 \mathrm{kHz}$, and 16,384 time samples were taken.

The Eulerian integral time scale for the longitudinal wind component $(\mathrm{u})$ was obtained by integrating over the autocorrelation function to the point where the autocorrelation first approached the zero point. A value of $0.36 \mathrm{~s}$ was obtained, which defines the time interval during which a substantial correlation exists between the velocity of different air parcels in the turbulent environment. The Eulerian integral length scale, which provides a measure of significant correlation for the spatial domain, can be obtained from the product of the mean longitudinal wind speed and the Eulerian integral time scale. A value of $0.18 \mathrm{~m}$ was obtained. The connection between the spatial and temporal aspects of turbulence depends on the acceptance of Taylor's frozen turbulence hypothesis, which assumes that turbulent eddies change in an imperceptible manner as they are carried by the mean wind past an in situ sensor. The integral length scale of the turbulence was approximately the same size as the characteristic dimension of the leaf. Thus, the leaf in the tunnel was subjected to energy-generating eddies of the same scale as it would be in a field setting.

Figure 2 is a plot of the smoothed spectrum presented in a manner consistent with meteorological usage. The product of frequency and the spectral estimate, $f S(f)$, has the dimensions of variance; when divided by the variance of the longitudinal wind component, it becomes dimensionless. Such spectra become smaller at both high and low frequencies. The horizontal axis is dimensionless frequency. In the present case, $N$ has the same numerical value as $f$. The theoretical slope for the high-frequency end of the spectrum is $N^{-2 / 3}(14)$. This portion of the spectrum is referred to as the inertial subrange. The region at lower frequencies is the energy-containing subrange, while the portion at higher frequencies is called the dissipation subrange (14). The inertial subrange gets its name from the fact that the dynamics are dominated by the inertial terms in the NavierStokes equation (these are all the terms 
except for the viscous and forcing terms). For the most part, the wind tunnel spectrum (Fig. 2) has a very limited inertial subrange. This deficiency is the result of low wind tunnel speeds and the narrow range of the size of the eddies. In the surface layer of the atmosphere, even though low wind speeds are observed, the range in the size of eddies is much greater, leading to the presence of an inertial subrange in the spectrum. See reference 14, pages 97104, for a detailed discussion of spectra and cospectra in plant canopies.

\section{RESULTS}

Experimental procedures. Live $N$. tabacum leaves were used for all flow visualization experiments. Artificial plants and leaves did not provide the natural motion required to study the turbulent flow fields used in this work. Plants were maintained in a growth chamber, and individual leaves were selected for mounting in the wind tunnel. Either the individual leaf was mounted on an artificial stem having height and diameter characteristics similar to the real stem, or a leaf attached to the real stem was used after removing the remaining leaves. When the real stem was used, the root system was left intact so that water could be supplied to the leaf to preserve its turgor and physical characteristics. The attached leaf method was adopted when tunnel residence time was long and water loss (and thus changes in leaf position, shape, orientation, and turgor) would become a problem. Each experiment was carried through to completion using the same live leaf. As described in previous sections, a laser light sheet in conjunction with the particles generated by a smoking wire was used to provide a visible flow field whose characteristics were captured on videotape. The smoking wire was located just upwind from the stem of the plant. This close proximity was required in order to provide sufficient quantities of smoke for the visualization process to work; however, the distance was sufficient to render heating/buoyancy effects insignificant.
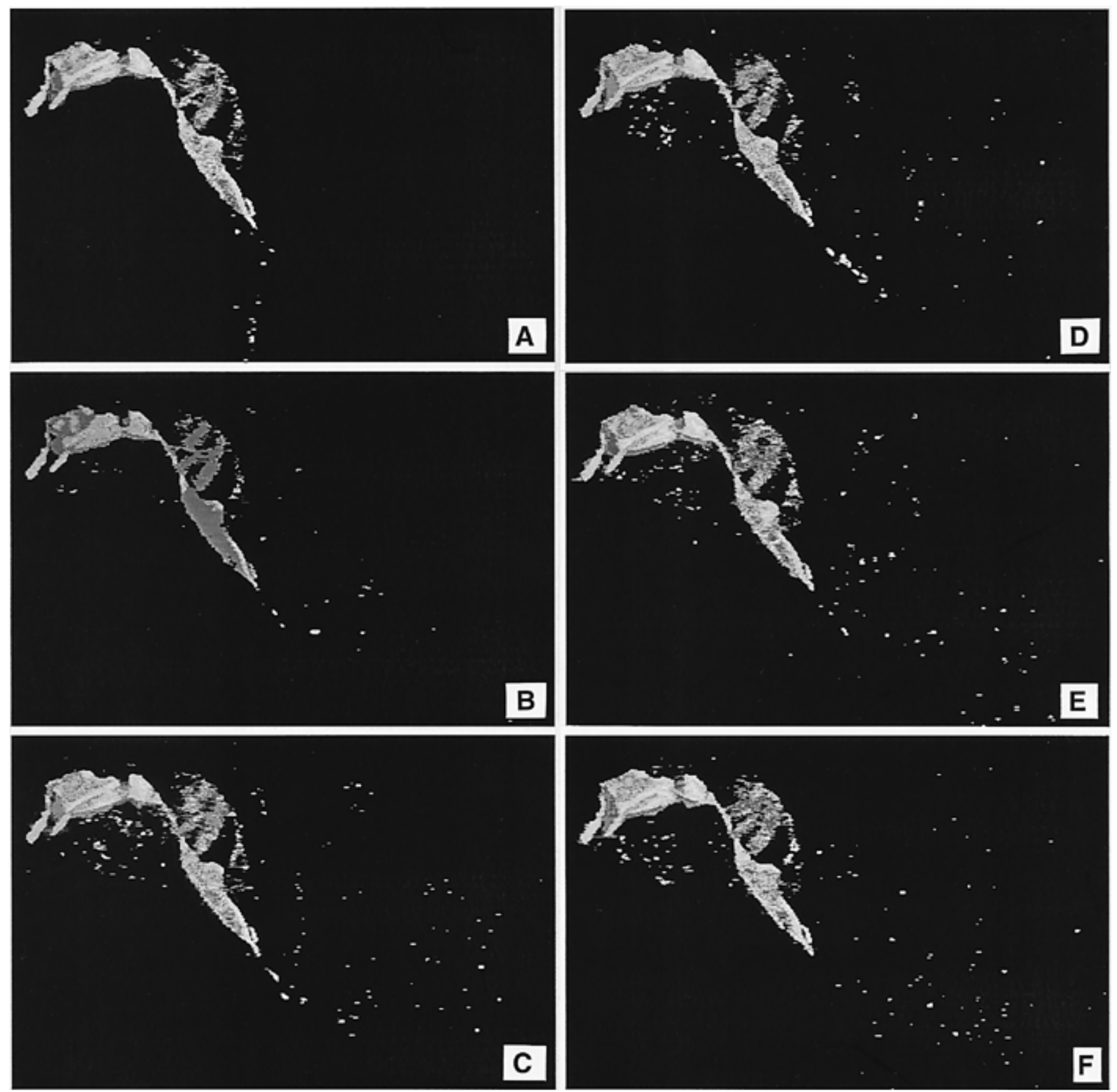

Fig. 4. Six images of spores leaving a diseased leaf. (A) Spore movement prior to the wind tunnel being turned on. (B) Spore movement immediately after the tunnel was turned on. (C to F) Spore movement spanning several seconds after the tunnel was turned on. 
Flow around leaves. Figure 3 is an artistic rendition of the vortices observed during the experiments. The flow fields are three-dimensional, and the indicated vortices constantly changed shape and shifted position. These vortices were clearly visible on the video tape. The existence of these regions and their relative strengths depend on many factors, which will be discussed in the following section. The vortex under the leaf seemed to be permanent and was present for both healthy and diseased leaves. The vortex on top of the leaf was most pronounced when the leaf had a major bend in it (which was true for many diseased leaves), and as a result, flow separation occurred (23). The structure of this vortex was constantly changing, and pieces of it were periodically shed downwind. The leaf tip vortex appeared to be present for both healthy and diseased leaves, and vortex shedding was also observed at the tip. The velocity measurements in the wake of an object such as a circular cylinder have a dominant periodicity which is easily observed at low Reynolds numbers. The frequency of this periodicity is expressed as a nondimensional parameter known as the Strouhal number. At high Reynolds numbers (above 5000), the periodicity in the wake becomes almost imperceptible and the wake may become completely turbulent. It remains to be determined if such coherent structures exist off the tip of a leaf.

The vortex on top of the leaf is the result of the phenomenon of flow separation. As fluid flow begins to occur over the smooth, curved surface of the leaf, the pressure gradient is negative (i.e., pressure decreases in the downwind direction) and the net pressure force on a boundary layer element over the leaf surface is in the forward (downwind) direction. The fluid finds this pressure gradient favorable since it

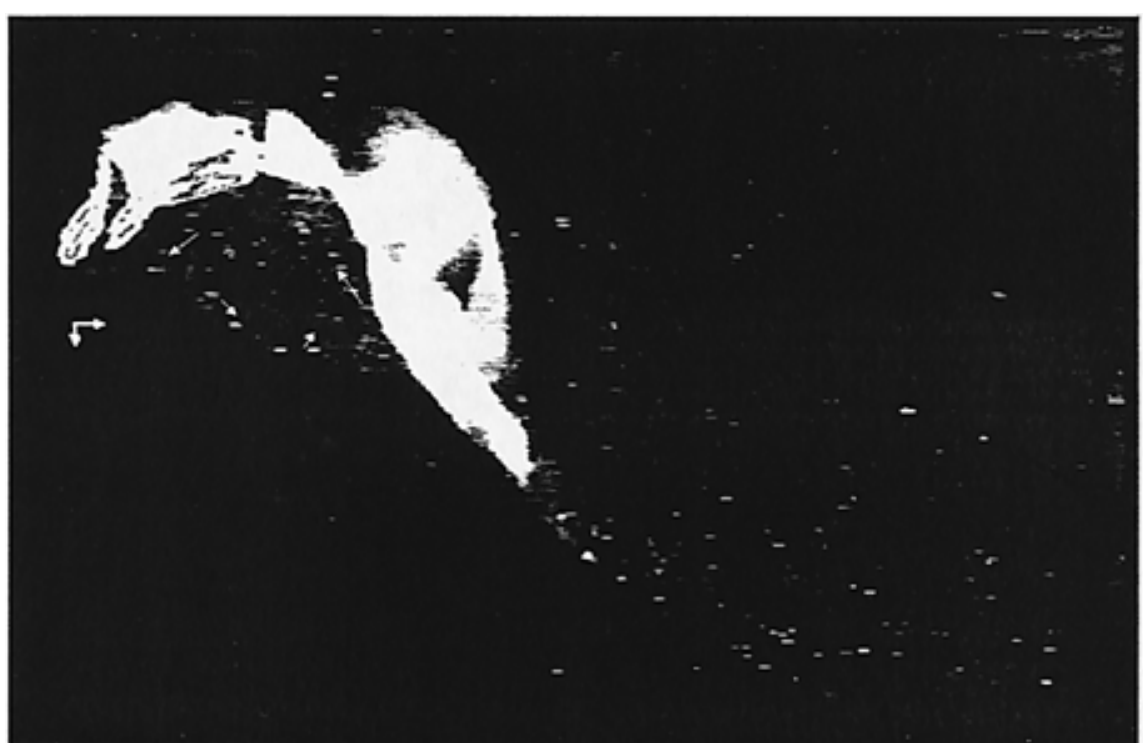

Fig. 5. Composite image showing the existence of the counterclockwise vortex on the upwind underside of the leaf. The arrows show the direction of spore movement.

tends to offset the effect of the boundary layer at the leaf's surface, which is to slow the fluid down. Beyond the bend in the leaf, pressure increases downwind, and thus the net pressure force tends to oppose the flow and serves to reduce the momentum of the fluid. At a point further downwind, fluid flow near the surface of the leaf ceases. Still further downwind, the flow close to the leaf surface is actually reversed. This flow reversal yields the vortex motion (recirculating flow) shown on the top side of the leaf. The leaf tip vortex is a result of flow separation initiated by fluid motion around the sharp corner provided by the leaf tip. The vortex under the leaf results from a split flow situation. As the fluid approaches the leaf, a portion goes over the upper surface while the rest goes under the leaf where, because of the bend in the leaf, it impacts at a high angle on the under surface of the leaf. Part of the fluid is turned counterclockwise under the leaf and sets up the observed vortex.

For the initial leaf, which was healthy, the stem was $47 \mathrm{~cm}$ long (from tunnel floor to interception of the leaf). The stem was located in the upwind direction, while the leaf tip was located in the downwind direction. In a wind tunnel setting, an infinite number of combinations of leaf and tunnel parameters is possible. The leaf position just described was used for the majority of the work. The distance from leaf tip to tunnel floor was $61 \mathrm{~cm}$ (as a leaf lost turgor, this number decreased). The characteristic dimension (length in the downwind direction) of the leaf was $32 \mathrm{~cm}$, while the projected (onto the longitudinal axis) dimension (since the leaf was curved) was 28 $\mathrm{cm}$. The Reynolds number at $0.5 \mathrm{~m} \mathrm{~s}^{-1}$ based on the leaf characteristic dimension is in the range of 5000 to 10000 . Flow around the healthy leaf exhibited some of the features shown in Figure 3. Because of

1062 Plant Disease / Vol. 81 No. 9 the shape of the leaf, the air generally tends to flow smoothly over the upper surface of the leaf, with no flow separation evident. When the leaf was drooping more from its midpoint on, flow separation on the upper surface was observed. The flow was generally composed of small-scale eddies characteristic of this level of turbulence. Events on the underside of the leaf were more interesting. At the leaf tip, there was evidence of the aforementioned counterclockwise vortex being generated and periodically shed downwind. Directly under the leaf, the flow appeared to split. Part of the flow proceeds in the downwind direction, moving slowly upward until it impacted on the leaf tip from underneath. Another portion of the flow participated in a counterclockwise elliptical vortex (with a major axis of about half the leaf characteristic dimension) beneath the upwind portion of the leaf. These two vortex motions are apparently permanent features of the flow field. The counterclockwise vortex under the upwind portion of the leaf provides a time-continuous mechanism for spore removal from a diseased leaf.

Niklas and his associates $(17,18)$ have used flow visualization techniques, computer simulation, and mathematical modeling to examine the flow patterns of pollen around parts of several plant species. A number of the flow features found in the current effort were also identified in their work.

Flow around diseased leaves. The flow visualization results presented in this section concern the flow around single diseased leaves. In all of the wind tunnel leaf tests, real attached spores were used. No attempt was made to use other aerosols to simulate the release and movement of spores from the leaf. Several problems arise when live spores are used. The main body of spores leaves the leaf in a relatively short time (a few minutes), thus limiting video recording time. Often, many sporulating leaves had to be mounted in the tunnel to obtain a leaf releasing adequate numbers of spores. The relative humidity in the tunnel averaged around 30\% during the experiment. The diseased leaves were turgid at tunnel temperature (around $23^{\circ} \mathrm{C}$ ). The vapor pressure gradient between the ambient air and the sporangiophores would have promoted the drying and twisting of the sporangiophores and thus the release of the spores.

Flow visualization techniques were again employed to examine the region around individual leaves. For diseased leaves, no smoke was generated. The scattered light came only from the spores themselves. Attempts to overlay smoke and spores in order to see gross flow features in addition to spore motion failed. The spores could not be detected within the smoke. The stem of the diseased leaf used was $38 \mathrm{~cm}$ long from the tunnel floor to the point where the leaf petiole started. The 
characteristic dimension of the diseased leaf was $39 \mathrm{~cm}$, while the projected dimension was $16 \mathrm{~cm}$ because of droop. The leaf tip was located $22 \mathrm{~cm}$ off the tunnel floor. The leaf tip pointed in the downwind direction in the tunnel.

The short time interval over which the spores were released required that the tunnel be operated at $0.5 \mathrm{~m} \mathrm{~s}^{-1}$. On the top side of the leaf, there was a clockwise rotating elliptic vortex (with a major axis equal to about one-third the leaf characteristic dimension) just downwind of the major bend in the leaf surface. The other two major vortices were also observed.

Figure 4 shows six enhanced images from the videotape recording of a diseased leaf. The bright pixels that appear in Figure
4 (and other figures showing spore motion) are digitized images of the spores. The original images were enhanced to produce spots large enough to remain visible following figure size reduction. The size of the spots does not represent actual spore size; it represents spore position. The video camera was mounted perpendicular to the downwind axis of the leaf. Figure 4A was recorded while the tunnel was turned off. Unfortunately, even when the tunnel motor/air movement system was off, the tunnel is still subject to vibrations and some air motion, which is evident in the figure. The tunnel is located near other large laboratories, which in conjunction with heating/air conditioning systems, produce lowlevel vibrations at all times. Thus, these figures are not truly indicative of totally still conditions.

The terminal settling velocity for the spores was estimated in the preliminary study mentioned above. An average value of $0.89 \mathrm{~cm} \mathrm{~s}^{-1}$ was determined for the velocity, which is close to the theoretical value provided by Stokes' Law.

Figure $4 \mathrm{~B}$ is an image taken immediately after the wind tunnel was turned on. The free stream centerline tunnel velocity was about $0.5 \mathrm{~m} \mathrm{~s}^{-1}$. In a companion study, the motion of the leaf tip at this tunnel setting was examined on videotape. The leaf proper and the leaf tip go through a complex, mainly vertical, oscillation sequence. The shedding of the vortices at the leaf tip may cause a forced oscillation of
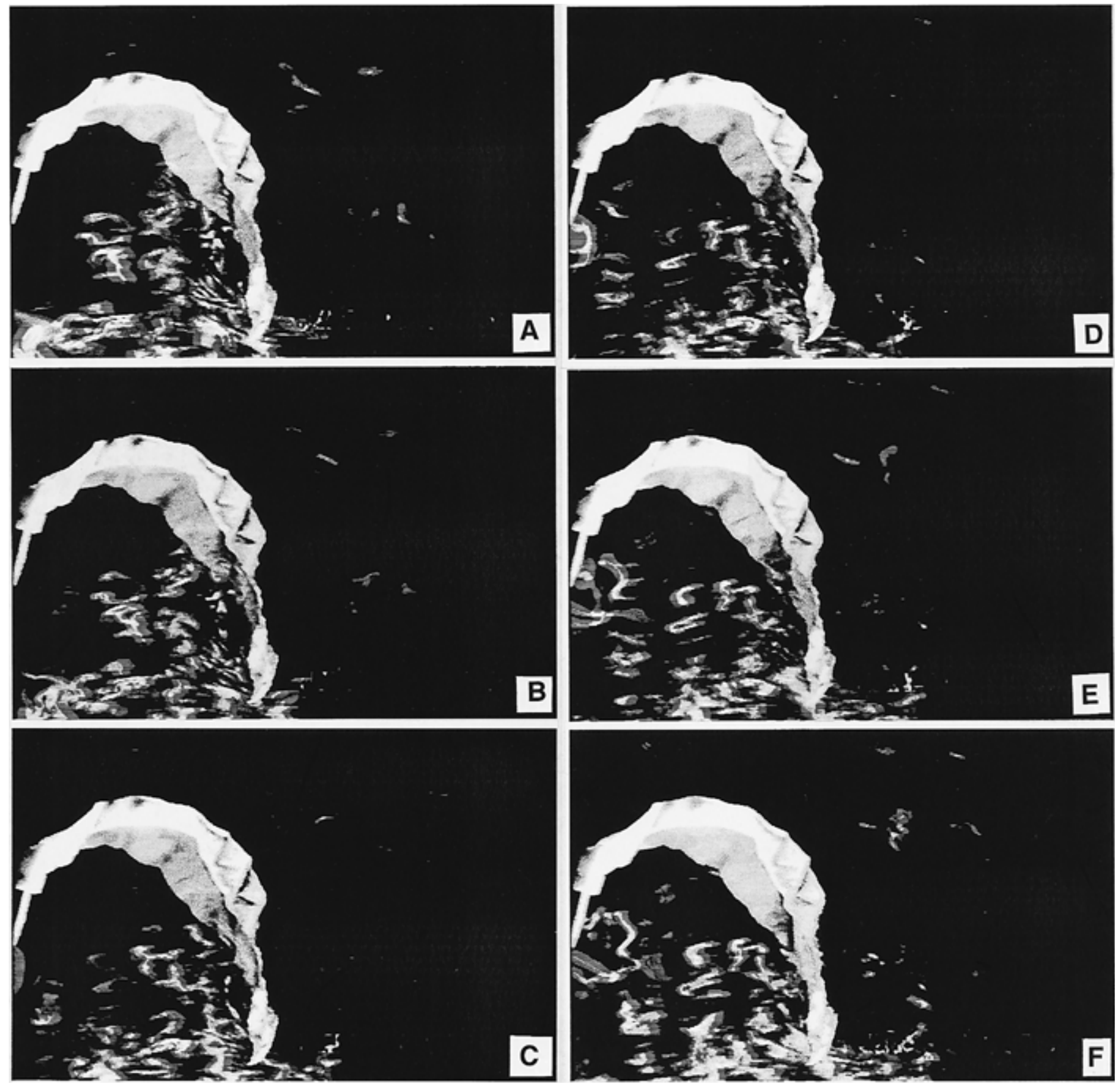

Fig. 6. Sequence of smoke-wire images showing the shedding of a vortex from the leaf tip. 
the leaf tip at the same frequency at which the vortices are being shed.

After tunnel motion had been initiated, there were two areas (as mentioned earlier) near the leaf that were of special interest. Just off the leaf tip, there was a counterclockwise vortex. Periodically these vortices are shed, i.e., they move downwind to be replaced by a new vortex. These vortices are responsible for the distribution of spores in front of the leaf, as shown in later images. Thus, the fluid motions cause a widespread distribution of the spores and aid in their escape from the canopy.

Another area of interest is just beneath the upwind part of the leaf near the stem. Flow around the stem itself would be similar to that around a two-dimensional cylinder in turbulent flow. Two recirculation zones would be expected to develop downwind of the stem centerline. However, of more interest from the disease standpoint is the counterclockwise vortex under the leaf in this region. The spores released into this vortex are clearly evident in Figure 4. This circulation pattern is not evident from the individual images. The video images indicate that this circulation is a permanent feature of the flow pattern; although its shape changes somewhat over time and spores leave the vortex to travel downwind under the leaf. Spores also move around the edges of the leaf to a position in front of the leaf. This persistent circulation helps, through mechanical action, to strip away the spores on the underside of the leaf in this area. The drag force (16) on the individual spore created by the flow in the vortex can be obtained from:

$$
F=C_{d} 0.5 \rho V^{2} A
$$

where the mean wind velocity $(V)$ is about $0.33 \mathrm{~m} \mathrm{~s}^{-1}$, the projected area of the spore (A) is about $3 \times 10^{-10} \mathrm{~m}^{2}$, the air density $(\rho)$ is $1.2 \mathrm{~kg} \mathrm{~m}^{-3}$, and the drag coefficient $\left(C_{d}\right)$ is 50 (for a sphere at a Reynolds number based on the sporangiospore, which is nearly spherical in shape) (16, page 111). The drag force is about $2 \times 10^{-9}$ $\mathrm{N}$. The Reynolds number based on spore diameter is less than 1. In this case, frictional drag and form drag are both important. This force appears to be enough to detach a spore from the stalk in the wind tunnel (1).

Despite the fact that the laser light sheet is only $1.5 \mathrm{~mm}$ thick, many spores remain in the light sheet from image to image. Using the software packages described above, one can determine the direction and speed of movement of individual spores and thus substantiate the existence of the vortex motion mentioned above.

Figure 5, which is a composite of several images, shows the existence of the counterclockwise vortex under the leaf. To ensure that the observed spores remained the same from image to image, which is required to make velocity measurements, spore pairs that maintained the same relative position to each other from image to image were selected when possible. This permanent vortex feature of the flow field has thus been sampled as conditions permit. The counterclockwise vortex can be identified using the arrows provided on the figure. Since the time between the adjacent images used to make up this figure was known, the spore speed can be calculated after calibrating the images for distance. The average speed of movement for the spores shown in Figure 5 is $0.33 \mathrm{~m} \mathrm{~s}^{-1}$. The PDA system was used to measure the wind speed at 10 positions in and around the vortex. The mean value of the magnitudes of the wind vectors at these points indicated that there was no significant difference between the wind speed and the speed of movement of the spores. These experimental results appeared to confirm the information given by the standard Stokes number and the turbulent version of the Stokes number (23, page 374), both of which had values less than 0.01 . When the Stokes number is sufficiently large, which this one is not, some eddies can project their spores out of the vortex, thereby enhancing the vortex exiting process. This enhanced projection of the spores out of the vortex can be assessed theoretically by calculating the turbulent Stokes number efficiency (23, page 375). For a value less than about 0.1 , the turbulent spore-containing eddy behaves like a single-phase fluid, i.e., the movement of the spores, despite their larger size, would be similar to that of the air molecules in the eddy, and the exiting enhancement would be minimal. In the present case, the calculated value for the efficiency was 0.26 . Thus, it appears that minimal exiting enhancement of the spores can be expected.

Figure 6 shows results that made use of the smoke generated by the smoke-wire technique to examine vortex shedding at the leaf tip. The first panel (A) shows smoke moving in a counterclockwise arc just beyond the leaf tip. Subsequent panels (B to F) show its downwind movement. Because of its lateral extent, the smoke curl does not move out of the laser light sheet as readily as the spores do. In subsequent panels (not shown), there is evidence of a new smoke curl developing at the leaf tip. It appeared that new smoke curls were being generated about every $0.25 \mathrm{~s}$.

Because this particular leaf has a large bend in it, flow separation is clearly visible on the videotape at the bend point on the upper leaf surface. A clockwise vortex is set up just downwind of the bend. This vortex is constantly changing shape and position, and portions of it are periodically shed downwind. There appears to be an interaction between this vortex and the aforementioned counterclockwise vortex just off the leaf tip. The combined action of these two vortices is to force spores to the upper leaf surface in that region where their flow patterns merge (see Figure 3). This could be considered an autoinfection process.

\section{DISCUSSION}

The turbulent flow in and around a diseased leaf clearly has many implications for the spread of plant pathogens. Caution must be exercised in the interpretation of these results, since only a single leaf has been considered. If one were considering this leaf as part of a whole plant located in a community of plants, the observed conditions would be considerably different. In such a setting, the intensity of turbulence would be greater in value and the mechanical interaction of adjacent leaves in the spore liberation process would need to be considered. The flow field around an individual leaf in this setting would be highly complicated. Early in the data collecting phase of the current project, an entire plant was placed in the tunnel. The flow field around the plant had many interesting features; however, because of the large size of the plant and the small cross-sectional area of the wind tunnel, boundary effects would bias the results. Nevertheless, the single leaf provides a reasonable beginning point for understanding spore release and movement.

The vortex beneath the upwind portion of the leaf (hereafter, vortex1), and the leaf tip vortex (vortex2) are important fluid dynamics mechanisms for spore separation from the leaf and movement away from the leaf. It is clear from the video imagery that within vortex 1 the drag force on the individual spore was sufficient to separate it from the stalk. The environmental conditions in the tunnel aided this separation by providing a constant steep vapor pressure gradient between the ambient air and the turgid leaf, which promoted stalk drying and twisting. The imagery clearly shows that the majority of the spores released in this portion of the leaf remain in vortex 1 and travel at a speed comparable to the wind speed. Stokes number calculations and efficiency calculations support these observed results. These counterclockwise moving spores make physical contact with spores that are still attached to their stalks and thus provide an additional means of spore liberation. In a more complex leaf/plant arrangement, additional physical mechanisms would be available for the mechanical release of the spores.

Smoke-wire results confirmed the existence of the counterclockwise leaf tip vortex. These smoke curls appeared to be shed downwind; however, the turbulent nature of the flow prevented the formation of well-defined and thus clearly visible structures. This vortex appears to play an important role in providing upward moving spores in front of the leaf. Presumably these spores would have the highest probability of escaping from the canopy. This vortex would also be a critical component 
(along with flow separation vortex on top of the leaf) in any autoinfection process.

The vortices identified in this study were all small. They were shown to be important in removing the spores from beneath the leaf and positioning them where larger scale eddies can bring about a mass exchange between the plant canopy and the lower atmosphere.

\section{LITERATURE CITED}

1. Aylor, D. E. 1975. Force required to detach conidia of Helminthosporium maydis. Plant Physiology, Lancaster 55:99-101.

2. Aylor, D. E. 1986. A framework for examining inter-regional aerial transport of fungal spores. Agric. For. Meteorol. 38:263-288.

3. Comte-Bellot, G. 1976. Hot-wire anemometry. Pages 209-231 in: Annual Review of Fluid Mechanics. M. Van Dyke, W. G. Vincenti, and J. V. Wehausen, eds. Annual Reviews, Palo Alto, CA.

4. Cornish, J. J. 1964. A device for the direct measurements of unsteady air flows and some characteristics of boundary layer transition. Mississippi State University, Aerophysics Research Note 24.

5. Davis, J. M. 1987. Modeling the long-range transport of plant pathogens in the atmosphere. Annu. Rev. Phytopathol. 25:169-188.

6. Davis, J. M., and Main, C. E. 1984. A regional analysis of the meteorological aspects of the spread and development of blue mold on tobacco. Boundary-Layer Meteorol. 28:271-304.

7. Davis, J. M., and Main, C. E. 1986. Applying atmospheric trajectory analysis to problems in epidemiology. Plant Dis. 70:490-497.

8. Davis, J. M., Main, C. E., and Nesmith, W. C. 1985. The biometeorology of blue mold of tobacco. Part II: The evidence for long-range sporangiospore transport. Pages 473-498 in: The Movement and Dispersal of Agricultur- ally Important Biotic Agents. D. R. MacKenzie, C. S. Barfield, G. C. Kennedy, and R. D. Berger, eds. Claitor's Publishing Division, Baton Rouge, LA.

9. Davis, J. M., Main, C. E., and Nesmith, W. C. 1989. The aerobiological aspects of the occurrence of blue mold in Kentucky in 1985. Pages 55-71 in: Blue Mold Diseases of Tobacco. C. E. Main and H. W. Spurr, eds. Proc. Symp. Blue Mold Dis. Tob.

10. Draxler, R. R. 1992. Hybrid single-particle Lagrangian integrated trajectories (HYSPLIT): Version 3.0-User's Guide and Model Description. NOAA Tech. Mem. ERL ARL-195. National Oceanic \& Atmospheric Administration, Silver Spring, MD.

11. Freymuth, P. 1978. A bibliography of thermal anemometer. TSI Quart. 4:2.

12. Heffter, J. L. 1980. Air Resources Laboratories Atmospheric Transport and Dispersion Model (ARL-ATAD), NOAA Tech. Mem. ERL ARL-81. National Oceanic \& Atmospheric Administration, Silver Spring, MD.

13. Heffter, J. L. 1983. Branching Atmospheric Trajectory (BAT) Model. NOAA Tech. Mem. ERL ARL-121. National Oceanic \& Atmospheric Administration, Silver Spring, MD.

14. Kaimal, J. C., and Finnigan, J. J. 1994. Atmospheric Boundary Layer Flows: Their Structure and Measurement. Oxford University Press, New York.

15. Leach, C. M. 1982. Active sporangium discharge by Peronospora destructor. Phytopathology 72:881-885.

16. Monteith, J. L., and Unsworth, M. H. 1990. Principles of Environmental Physics. Edward Arnold, London.

17. Niklas, K. J. 1985. The aerodynamics of wind pollination. Bot. Rev. 51:328-386.

18. Niklas, K. J., and Buchmann, S. L. 1988. Aerobiology and pollen capture of orchardgrown Pistacia vera (Anacardiaceae). Am. J. Bot. 75:1813-1829.

19. Pankhurst, R. C., and Holder, D. W. 1952.
Wind-Tunnel Techniques: An Account of Experimental Methods in Low- and High-Speed Wind Tunnels. Sir Isaac Pitman \& Sons, LTD, London.

20. Panofsky, H. A., and Dutton, J. A. 1984. Atmospheric Turbulence: Models and Methods for Engineering Applications. John Wiley $\&$ Sons, New York.

21. Pasquill, F., and Smith, F. B. 1983. Atmospheric Diffusion. Halsted Press, New York, 437pp.

22. Pinckard, J. A. 1942. The mechanism of spore dispersal in Peronospora tabacina and certain other downy mildew fungi. Phytopathology 32:505-511.

23. Rosner, D. E. 1986. Transport processes in chemically reacting flow systems. Butterworths, Boston.

24. Sorbjan, Z. 1989. The structure of the atmospheric boundary layer. Prentice Hall, Englewood Cliffs, NJ.

25. Stull, R. B. 1988. An Introduction to Boundary Layer Meteorology. Kluwer Academic Publishers, Boston.

26. Van de Hulst, H. C. 1957. Light Scattering by Small Particles. John Wiley \& Sons, New York.

27. Vanderpool, R. W., and Peters, T. M. 1994 Wind tunnel evaluation of the RAAMP sampler. Technical Report, Research Triangle Institute, Research Triangle Park, NC.

28. Yamada, H. 1973. Instantaneous measurements of air flows by smoke-wire technique. Trans. Jpn. Mech. Eng. 39:726.

29. Yao, C., Arya, S. P., Davis, J. M., and Main, C. E. 1997. A numerical model of the transport and diffusion of Peronospora tabacina spores in the evolving atmospheric boundary layer. Atmospheric Environment. 31:17091714

30. Zare, M., and Durst, F. 1993. Laser Doppler measurements in two-phase flows. Pages 428452 in: Selected Papers on Laser Doppler Velocimetry. R. J. Adrian, ed. SPIE Optical Engineering Press, Bellingham, WA. 